\title{
SALUD MENTAL EN EL ADULTO MAYOR: TRASTORNOS NEUROCOGNITIVOS MAYORES, AFECTIVOS Y DEL SUEÑO
}

\author{
Tania Tello-Rodríguez ${ }^{1,2, a}$, Renato D. Alarcón ${ }^{2,3, b}$, Darwin Vizcarra-Escobar 2,4,c
}

\begin{abstract}
RESUMEN
Existen numerosos factores biológicos, psicológicos y sociales con impacto más o menos prominente en la salud mental de las personas adultas mayores. Aparte de componentes derivados de los procesos normales de envejecimiento o de la coocurrencia de enfermedades médicas diversas, eventos como la muerte de un ser querido, la jubilación o la discapacidad, contribuyen significativamente a una variedad de problemas mentales o emocionales en esta fase del ciclo vital. Los problemas más frecuentes afectan las esferas neurocognitiva, afectiva y onírica. Los trastornos neurocognitivos mayores reducen el rendimiento general del paciente y generan con ello exigentes necesidades de dependencia y cuidado cercano. Los trastornos afectivos pueden acentuarse por falta de apoyo familiar y disminución marcada de interacciones sociales que pueden dar lugar a un significativo aislamiento con conducta suicida resultante. La mayor frecuencia de trastornos del sueño como insomnio, somnolencia diurna y trastornos específicos como apnea obstructiva alteran significativamente la calidad de vida de esta población.
\end{abstract}

Palabras clave: Salud mental; Adulto mayor; Trastornos neurocognitivos; Trastornos del sueño; Depresión (fuente: Decs BIREME).

\section{MENTAL HEALTH IN OLDER ADULTS: MAJOR NEUROCOGNITIVE, AFFECTIVE, AND SLEEP DISORDERS}

\begin{abstract}
Numerous biological, psychological, and social factors influence the mental health of elderly individuals to varying degrees. Apart from components related to the normal aging process and the co-occurrence of various medical conditions, events such as the death of a loved one, retirement, or disability significantly contribute to a variety of mental and emotional problems in this stage of the life cycle. The most frequent problems affect the neurocognitive, emotional, and oneiric spheres. Major neurocognitive disorders reduce one's overall performance and, thus, increase their need for close care. Affective disorders may be exacerbated by the lack of family support and decreased social interactions, which may lead to significant isolation result in suicidal behavior. The increased frequency of sleep disorders such as insomnia and daytime sleepiness and specific disorders such as obstructive apnea significantly alter the quality of life of this population.
\end{abstract}

Key Words: Mental Health; Elderly; Neurocognitive disorders; Sleep disorders; Depression (source: MeSH NLM).

\section{INTRODUCCIÓN}

Los problemas de salud mental son frecuentes en la población adulta mayor (AM): más de un $20 \%$ de éste grupo etario puede padecerlos con variados grados de severidad, de acuerdo con numerosos estudios epidemiológicos a nivel mundial (1). Debe pues prestárseles tanta o más importancia que a problemas de salud física ya que pueden exacerbar sus manifestaciones (en particular las de enfermedades crónicas) e incrementar la dependencia funcional y el mayor uso de los recursos sanitarios. Por otro lado, los problemas de salud mental muchas veces pasan desapercibidos, no son diagnosticados o son subtratados, por lo que la capacitación de los profesionales en esta área es indispensable para un manejo oportuno y eficiente. Es necesario, además, contar con políticas, estrategias y servicios especializados en salud mental para este grupo etario, de modo tal que su calidad de vida y la de sus familiares pueda ser preservada ${ }^{(1)}$.

\footnotetext{
Instituto de Gerontología, Universidad Peruana Cayetano Heredia. Lima, Perú.

Facultad de Medicina, Universidad Peruana Cayetano Heredia. Lima, Perú.

Mayo Clinic College of Medicine. Rochester. Minnesota, EE.UU.

Hypnos, Instituto del Sueño, Clínica San Felipe. Lima, Perú.

a Médico geriatra, magíster en Geriatría y Gerontología, ${ }^{b}$ médico psiquiatra, magíster en Salud Pública, ${ }^{c}$ médico neurólogo, especialista en Medicina del Sueño. Recibido: 07/03/2016 Aprobado: 01/06/2016
}

Citar como: Tello-Rodríguez T, Alarcón RD, Vizcarra-Escobar D. Salud mental en el adulto mayor: trastornos neurocognitivos mayores, afectivos y del sueño. Rev Peru Med Exp Salud Publica. 2016;33(2):342-50. doi: 10.17843/rpmesp.2016.332.2211 
Se describen a continuación tres grupos de problemas de salud mental que repercuten notablemente en el devenir vital de los adultos mayores: trastornos neurocognitivos mayores, problemas afectivos o del ánimo y desórdenes del sueño.

\section{TRASTORNOS NEUROCOGNITIVOS MAYORES (DEMENCIA)}

Trastorno neurocognitivo mayor (TNCM) es la nueva denominación con que la quinta versión del Manual Diagnóstico y Estadístico de Trastornos Mentales (DSM5) de la Asociación Psiquiátrica Americana (APA), ha sustituido al término demencia utilizado previamente ${ }^{(2)}$.

Existe evidencia de que las cifras de la población de adultos mayores a nivel mundial y en nuestro país están aumentando a un ritmo acelerado, debido, en parte, a la mejoría de la atención médica global; a su vez, la subpoblación que más crece es la de personas mayores de 80 años, es decir aquellas en las que el riesgo de presentar demencia está acentuado: se presume, entonces, que con ello, la prevalencia del TNCM se incrementará. El deterioro de la cognición y las concomitantes alteraciones funcionales y conductuales hacen que pacientes de este tipo sean cada vez más dependientes de los cuidadores en un proceso cuyo impacto en la familia y en su entorno social y laboral es intensamente negativo ${ }^{(3,4)}$.

\section{EPIDEMIOLOGÍA}

La prevalencia de demencia antes de los 65 años es de entre 2 a $10 \%$, pero ella se duplica con cada intervalo de 5 años después de los 65 años de edad ${ }^{(1,3,5)}$. En 2011 se calculó que, a nivel mundial, 35 millones de personas padecían de demencia y diversos estudios epidemiológicos indican que la cifra se duplicará cada 20 años. La demencia es una de las principales causas de discapacidad y genera un alto costo social; se le atribuye $11,9 \%$ de los años vividos con discapacidad a causa de una enfermedad no transmisible ${ }^{(5,6)}$.

En estudios locales se ha encontrado que la prevalencia de demencia en Lima fue de 9,3\% (7). Custodio et al. detectaron un $6,85 \%$, siendo el alzhéimer el diagnóstico más frecuente $(56,2 \%)$ que aumenta con la edad y ocurre con mayor frecuencia en pacientes de sexo femenino ${ }^{\left({ }^{8}\right)}$. En el estudio realizado por el Instituto Nacional de Salud Mental en Lima se encontró una prevalencia de $6,7 \%{ }^{(9)}$.

\section{ETIOPATOGENIA}

Dentro de las principales causas de demencia (que operan también como definitorias de etiquetas subdiagnósticas) se cuentan el alzhéimer con una variedad de alteraciones morfofuncionales en varias regiones cerebrales, constituyendo el 60 a $80 \%$ de los casos; demencia vascular (20 a $40 \%$ ); demencia por enfermedad debida a cuerpos de Lewy (5 a 20\%) y demencia fronto-temporal (5 a 20\%) ${ }^{(3)}$.

Dentro de los factores de riesgo se han señalado los de carácter genético (APOE 4), sociodemográfico (edad, raza, educación, historia familiar), vasculares o metabólicos (hipertensión, hiperlipidemia, diabetes), trauma cerebral, estilos de vida o hábitos (dietas irregulares, obesidad, consumo de tabaco o alcohol, etc. $)^{(3)}$.

\section{EVALUACIÓN, CRITERIOS DIAGNÓSTICOS Y CLASIFICACIÓN}

La evaluación del paciente con sospecha de demencia debe basarse en una cuidadosa información anamnésica recogida del propio paciente, familiares o cuidadores, con énfasis en la exploración de cambios cognitivos, funcionales y de conducta. A su vez, la exploración física debe incluir un adecuado examen neurológico, evaluación funcional motora y exámenes auxiliares que incluyan vitamina B12, hormonas tiroideas y estudios de neuroimágenes. La Tabla 1 muestra los criterios diagnósticos de TNCM (demencia) incluidos en el DSM-5 (2).

El trastorno neurocognitivo mayor se diferencia del trastorno neurocognitivo menor porque en el primero las dificultades cognitivas alteran la capacidad de la persona para llevar a cabo actividades de la vida diaria ${ }^{(2,10)}$. El TNCM puede ser clasificado en: 1) Leve, si presenta dificultades en las actividades instrumentales de la vida diaria (por ejm., comprar, hablar por teléfono, tomar un bus, cocinar, etc.); 2) Moderado, cuando ocurren alteraciones en las actividades

Tabla 1. Criterios diagnósticos propuestos en el DSM-5 para trastorno neurocognitivo mayor*.

\section{Criterios diagnósticos}

a. Evidencia de una sustancial declinación cognitiva de un nivel previo de mayor desempeño en uno o más de los dominios cognitivos (aprendizaje y memoria, atención compleja, función ejecutiva, lenguaje, habilidad perceptual motora o cognición social).

b. Los déficits cognitivos son suficientes para interferir con la independencia del paciente (por ejemplo, requieren asistencia para las actividades instrumentales de la vida diaria, tareas complejas como manejo de la medicación o dinero).

c. Los déficits cognitivos no ocurren exclusivamente en el contexto de un delirio.

d. Los déficits cognitivos no son atribuibles de forma primaria a la presencia de otros trastornos mentales (ejemplo: trastorno depresivo mayor, esquizofrenia).

*American Psychiatric Association. Diagnostic and Statistical Manual of Mental Disorders, 5th. Edition (DSM-5). Washington DC: American Psychiatric Publishing; 2013 
básicas de la vida diaria (por ejm., alimentarse, vestirse, bañarse, caminar, control de esfínteres, etc.), y 3) Grave, cuando la persona es totalmente dependiente de otros para la ejecución de actividades básicas de la vida diaria ${ }^{(2,10)}$.

\section{PRUEBAS COGNITIVAS}

El MiniMental Status Examination (MMSE) y otras pruebas de cribado para demencia tienen una sensibilidad de 75 a $92 \%$ y una especificidad de 81 a $91 \%$. El MMSE es el más ampliamente estudiado, un puntaje menor a 24 es sugestivo de demencia; se reconoce que los valores pueden ser influenciados por edad, grado de instrucción, dominio del lenguaje, compromiso visual, etc. ${ }^{(11)}$.

Otro test, el Mini-cog consta de dos componentes: recuerdo de tres ítems y la prueba del dibujo de un reloj; tiene una sensibilidad de 76 a $99 \%$ y especificidad 89 a $96 \%$. En un estudio realizado en nuestro país sobre la validación de la prueba de dibujo del reloj (versión de manos) como prueba de despistaje para detectar demencia en una población adulta mayor, se encontró que un punto de corte de 7 tiene una sensibilidad de $99 \%$ y una especificidad de $83 \%$ (12). Los test neuropsicológicos permiten además evaluar los perfiles cognitivos de cada tipo de demencia.

\section{TRATAMIENTO}

En diversos ensayos clínicos para el manejo de la enfermedad de Alzheimer se ha visto que los beneficios son modestos en cuanto al retraso del deterioro cognitivo o funcional y a la disminución de síntomas neuropsiquiátricos ${ }^{(13)}$. Es necesario que los médicos, la familia o cuidadores y los pacientes mismos, de ser posible, deban discutir cuáles son las metas cognitivas, funcionales y conductuales antes de iniciar un tratamiento farmacológico. Si se decide utilizar, por ejemplo, inhibidores de colinesterasa y los objetivos del tratamiento no se logran después de un tiempo razonable (alrededor de 12 semanas), puede decidirse la suspensión del tratamiento, tomándose en cuenta que los riesgos y beneficios de la terapia a largo plazo no han sido bien establecidas. Por otro lado, el tratamiento no farmacológico es muy importante: la educación y capacitación del cuidador en la atención y prevención de riesgos del paciente con demencia tiene una importancia crítica. Un manejo básico y preventivo de los trastornos de conducta, una adecuada dieta y programa de ejercicios y actividades físicas, terapia ocupacional y cognitiva, así como la planificación de los cuidados en etapas terminales del cuadro clínico, son componentes fundamentales de este manejo. Es importante, además, organizar a la familia en una cooperación adecuada en la atención del paciente a fin de evitar sobrecargas de la labor del cuidador ${ }^{(13)}$.

\section{TRASTORNOS AFECTIVOS EN EL ADULTO MAYOR}

El periodo del ciclo vital conocido como "adultez mayor" o senescencia, posee probablemente características de mayor singularidad que las de otras fases, al acumular factores y experiencias de etapas previas y afrontar desenlaces que no por previsibles o conocidos dejan de generar ansiedad o expectativas de duda, conflicto, pesimismo e incertidumbre. El componente afectivo o emocional de la vida cuotidiana del adulto mayor, muchas veces privada del calor y el apoyo del grupo familiar o de un mínimo de interacciones sociales positivas, confiere significados diferentes a la gradual reducción de diversas funciones biológicas, cognitivas o sensoriales y, con ella, una mayor vulnerabilidad a variados agentes o factores patógenos ${ }^{(14)}$. Finalmente, la psicopatología o clínica psicogeriátrica, aun cuando utilizando etiquetas nosológicas similares a aquellas de fases previas del ciclo vital, entraña rasgos distintivos que es conveniente conocer y delinear claramente a fin de proveer la atención profesional más apropiada y oportuna ${ }^{(15)}$.

\section{PERSPECTIVAS GENERALES}

Los trastornos afectivos, en general, cubren en los sistemas nosológicos más recientes, hasta dos áreas fundamentales: trastornos depresivos propiamente tales y trastornos bipolares (2). Queda claro que otros cuadros psiquiátricos, tanto en adultos como en ancianos, pueden presentar un definido componente afectivo: es el caso de trastornos ansiosos, obsesivo-compulsivos, relacionados con trauma y estresores, trastornos neurocognitivos, de personalidad y adictivos. Estudios neurobiológicos muestran desactivación funcional en las regiones parietal y frontolateral del cerebro, con pérdida de materia blanca en el giro parahipocampo ${ }^{(16)}$. La responsabilidad del médico de atención primaria, así como la del médico geriatra, abarca no solo la necesidad de detectar tal componente, sino la de precisar sus rasgos de entidad clínica autónoma (por ejemplo, depresión mayor), de manifestación comórbida (vgr., síntomas depresivos en un incipiente cuadro demencial), un síndrome reactivo o una respuesta al padecimiento de un cuadro médico incipiente o más o menos definido.

El hallazgo de síntomas afectivos (principalmente depresivos) en el adulto mayor puede perder significación si el médico los ubica en el contexto de un cuadro físico o los "explica" como respuesta "normal" a experiencias de pérdida o envejecimiento ${ }^{(17)}$. La evaluación de este tipo de síntomas se complica en función de severidad variable en el curso de días o semanas, combinaciones sindrómicas variadas, duración y evolución impredecibles. Si a ello se une información insuficiente por deficiencias del propio paciente o de su entorno sociofamiliar, la tarea clínica puede ser difícil y hasta frustrante. 


\section{ENTIDADES ESPECÍFICAS}

Trastorno depresivo mayor (TDM). Se considera que hasta un $2 \%$ de la población adulta mayor puede experimentar TDM, las mujeres representan más de la mitad de esta cifra ${ }^{(18)}$. Los criterios diagnósticos más aceptados en esta categoría diagnóstica, cuandoaplicada a adultos mayores, incluyen persistentes sentimientos de tristeza, vacío emocional, desesperanza, anhedonia, apatía, insomnia o hipersomnia, retardo psicomotor, aislamiento social y disminución de funciones cognitivas. En ancianos así afectados, la fragilidad física se hace más evidente, por lo que es importante atribuir correctamente a TDM síntomas físicos tales como dolores difusos, fatiga y constipación. Muchos ancianos con TDM permanecen en cama largas horas del día y pueden exhibir dependencia y negativismo que, en casos, alcanza niveles psicóticos. El deterioro funcional puede ser similar al que se observa en enfermedades médicas graves. Anomalías estructurales en el giro cingulado subgenual y el fascículo uncinado perpetúan estados de apatía al interferir con la estimulación cortical prefrontal de la actividad límbica ${ }^{(16)}$.

Trastorno depresivo persistente (distimia). También llamada "depresión crónica" o "depresión menor", la diferencia fundamental con TDM es el menor grado de severidad, aun cuando el impacto en la vida del paciente puede ser igualmente importante ${ }^{(2,19)}$. Los síntomas pueden estar presentes por meses y hasta años y su distinción con otros cuadros psiquiátricos o, más aun, con entidades médicas tales como hipotiroidismo, es fundamental. La distimia puede también sobrevenir acompañada por distrés ansioso, rasgos melancólicos o los llamados rasgos atípicos tales como disturbios alimentarios y sueño irregular. Los ancianos son también más propensos a cuadros de duelo prolongado cuya ocurrencia "en ondas" y una duración mayor de 7-10 meses, con sentimientos de culpa, desaliento, ideas de muerte o suicidio, pueden anunciar una transición definida hacia el TDM.

Conducta suicida en ancianos. Mujeres de 60 años, o más, presentan un índice más alto de intentos suicidas que su contraparte masculina, pero la prevalencia de suicidios consumados es más alta entre los varones, llegando a ser hasta dos veces mayor que la de la población general. Aparte de sexo masculino, los factores específicos de riesgo incluyen presencia de comorbilidades médicas, contacto interpersonal pobre, duelo prolongado, muerte reciente de un ser querido, intento suicida previo o problemas financieros ${ }^{(20)}$. La modalidad escogida puede ser fundamentalmente sobredosis medicamentosa, salto a abismos o autoagresión con armas punzocortantes en poblaciones latinoamericanas (21) y uso de armas de fuego en Norteamérica o Europa (14). En muchos de estos pacientes no ha habido un diagnóstico formal previo de depresión, pero sí se registran visitas médicas más o menos frecuentes en semanas previas al intento suicida.

Depresión geriátrica y comorbilidades médicas. La alta mortalidad en pacientes geriátricos con depresión concomitante es un fenómeno reconocido. En muchos casos, médicos de atención primaria son los primeros expuestos al contacto con estos pacientes, de modo tal que la coexistencia de depresión con diagnósticos tales como accidentes cerebrovasculares, cáncer, enfermedades respiratorias, diabetes y artritis, genera significativas interacciones clínicas. El infarto miocárdico y/o enfermedad isquémica son casi inmediatamente seguidas por depresión marcada y muerte. Trastornos neurológicos tales como enfermedad de Parkinson y esclerosis múltiple, enfermedades endocrinas (vgr. hipotiroidismo), déficits vitamínicos (B12, D y K), nefropatías, enfermedad cardiaca congestiva y cáncer de páncreas pueden debutar con síntomas depresivos, demandando por ello especial atención y alerta continua por parte de los médicos y geriatras tratantes.

Depresión y déficits cognitivos. Una depresión severa puede afectar seriamente la función cognitiva de pacientes ancianos y acompañar incluso a trastornos neurocognitivos mayor (demencia) o menor. Ocasionalmente, se utiliza el término "pseudodemencia" para caracterizar estos casos. Apatía, síntoma común de demencia, puede, sin embargo, no ser parte del cuadro de fondo y constituirse, por lo tanto, en entidad manejable. La llamada "depresión de inicio tardío" puede ser un indicador temprano de enfermedad de Alzheimer, aunque también ha sido vinculada a cuadros cerebrovasculares en el contexto de la llamada hipótesis de "depresión vascular" (22).

Trastorno bipolar (TB) y relacionados. De ocurrencia predominante en poblaciones jóvenes y con edad de inicio más frecuente en la segunda o tercera década de la vida, el TB presenta, sin embargo, otro desafío clínico en su versión geriátrica ${ }^{(23)}$. Se reconoce hoy una tendencia creciente de prevalencia (hasta el 10\% en algunos estudios) ${ }^{(24)}$, una lista mayor de trastornos médicos (neurológicos, endocrinos, farmacológicos y cognitivos, en particular) como precipitantes o comórbidos (más del $70 \%$ ) y la compleja gama de entidades y características relacionadas al llamado espectro bipolar (variedades de bipolaridad, cicladores rápidos, presencia de catatonía, patrón estacional, rasgos atípicos o mixtos, etc.). Predominan episodios depresivos con rasgos melancólicos y pacientes mujeres parecen mostrar incidencia más alta de ciclaje rápido y disforia durante episodios maniacos ${ }^{(23,24)}$. Los episodios pueden ser más frecuentes y de duración más corta, configurando numerosas recurrencias. 


\section{TRATAMIENTO Y PRONÓSTICO}

Existe acuerdo en que el mejor enfoque terapéutico es la adecuada combinación de estrategias psicofarmacológicas y psicosociales. Este tipo de manejo reduce niveles de morbilidad y mortalidad, así como exigencias o derrotismo por parte de familiares, agencias de salud y servicios sociales.

\begin{abstract}
Afronte psicofarmacológico. La efectividad del manejo farmacológico de la depresión $(60-80 \%)$ no es sustancialmente afectada por el factor edad (25). En adultos mayores, por lo tanto, pasos específicos tales como diagnóstico certero, elección adecuada de la medicación, administración gradual del fármaco y seguimiento y duración apropiada del tratamiento, mejorarán el desenlace clínico. Sin embargo, solo la mitad de los pacientes responde al primer intento, la respuesta toma tiempo más largo que las habituales tres o cuatro semanas, y es preferible una actitud conservadora más que agresiva en el uso de combinaciones medicamentosas. La adherencia al tratamiento es, a veces, problemática. Se recomienda el uso de inhibidores selectivos de la recaptación de serotonina (ISRS) iniciando el tratamiento con la mitad de la dosis habitual. Las benzodiacepinas y tranquilizantes mayores pueden ser útiles en casos con ansiedad concomitante, pero su uso debe ser limitado en dosis y tiempo de administración ya que hipersomnia y fatiga pueden ser efectos secundarios importantes. En casos de manía o hipomanía, el litio continúa siendo el medicamento más efectivo cuando es usado con precaución y tino.
\end{abstract}

Afronte psicosocioterapéutico. Terapia cognitivoconductual, psicoterapia interpersonal, terapia reminiscente y terapia de adaptación a problemas son las modalidades más frecuentemente utilizadas ${ }^{(26)}$. La participación de familiares y otras personas cercanas es crucial. Otros enfoques tales como terapia musical y actividad física regulada son complementos útiles ${ }^{(27)}$.

Otros tratamientos. La terapia electroconvulsiva (ECT) continúa siendo un instrumento útil en el manejo de depresión geriátrica severa, en la llamada depresión refractaria, en cuadros bipolares con síntomas catatónicosy/o psicóticos, agitación psicomotriz, anorexia o ideación suicida prominente. En casos conocidos puede ser necesario administrar la ECT antes que otro ensayo farmacológico, pero el número de aplicaciones no debe ser mayor de 12. La Terapia de mantenimiento (semanal primero, mensual después) viene ganando aceptación. Los efectos cognitivos (amnesia retrógrada y anterógrada) son factores determinantes en el número y frecuencia de administración de la ECT (28)

Finalmente, otras técnicas de tratamiento somático tales como estimulación magnética transcraneal (TMS), estimulación con corriente directa transcraneal (tDCS) y estimulación cerebral profunda (DBS) están aún en fases estimativas en poblaciones geriátricas ${ }^{(29)}$.

\section{TRASTORNOS DEL SUEÑO EN ADULTO MAYOR}

Los trastornos del sueño se consideran un problema de salud pública. Prevalencias rampantes de insomnio, somnolencia diurna y trastornos específicos como apnea obstructiva del sueño (SAOS) así lo demuestran.

Los llamados estados de conciencia son tres: vigilia, sueño REM y sueño no REM; el último se subdivide en tres subestadios (N1, N2, N3), cuyo número crece en función del enlentecimiento del electroencefalograma y la resistencia a ser despertado. Cambios fisiológicos asociados al envejecimiento involucran la disminución del sueño N3 y el incremento en el número de despertares. El sueño REM, de presentación periódica a lo largo de la noche, disminuye con el envejecimiento, aunque en una proporción menor que N3. Las necesidades de sueño del adulto mayor parecen ser semejantes a las del adulto en edad media; la dificultad para poder mantener un sueño consistente en la noche incrementa el tiempo de estancia en cama, el tiempo que transcurre hasta iniciar el sueño, los despertares, el tiempo de vigilia nocturna y las siestas durante el día.

Existen, asimismo, procesos reguladores del sueño: el proceso homeostático, merced al cual la extensión de la vigilia aumenta la presión por dormir, disminuye, sin embargo, su eficiencia con el envejecimiento. El segundo, el proceso circadiano, que condiciona actividad diurna y sueño nocturno, tiende a preservarse un tanto mejor.

\section{LAS FUNCIONES DEL SUEÑO}

Son diversas y llevan a un buen proceso de adaptación. La recuperación de energía es parte integral del proceso homeostático. El no dormir bien está asociado a dolor corporal, patología que crece con la edad. El sueño favorece la liberación de hormonas anabólicas, y su disminución está asociada con el proceso de envejecimiento. Niveles de adrenalina de la vigilia, disminuyen por el predominio colinérgico durante el sueño, la perturbación del sueño afectaría la presión arterial, el ritmo cardiaco, y sus efectos sobre la circulación. La atención, y memoria de corto plazo pueden verse comprometidas por problemas de sueño ${ }^{(30)}$. SAOS se asocia con microinfartos cerebrales, riesgo de ictus y deterioro cognitivo secundario ${ }^{(31)}$. Se viene trabajando la hipótesis de una mayor expresión de genes ligados a enfermedad de Alzheimerfacilitada por SAOS ${ }^{\left({ }^{(2)}\right)}$. Igualmente, los trastornos del sueño incrementan la fragilidad global del anciano. 


\section{ENTIDADES ESPECÍFICAS}

Insomnio. La prevalencia de insomnio en adultos mayores se encuentra entre 13 a $47 \%{ }^{(33)}$. En el adulto mayor los mecanismos de hiperalerta nocturna, relacionados con trastornos del ánimo, problemas con higiene del sueño(Tabla 2) y condicionamiento a la hipervigilancia, son semejantes a las del adulto joven; sin embargo los trastornos médicos adquieren mayor relevancia, destacando los efectos secundarios de medicación y los trastornos ocultos del sueño como SAOS y el síndrome de piernas inquietas (SPI).

Desde el punto de vista psicoterapéutico, la terapia cognitivo-conductual muestra un buen desempeño en el $A M$ al igual que en el adulto joven ${ }^{(32)}$. Algunos fármacos han sido probados en AM (32): agonistas de benzodiacepinas, como zolpidem y eszoplicona, benzodiazepinas de acción corta como el triazolam. Es necesario ajustar dosis en función de edad, estatus hepático y renal ${ }^{(32)}$. No se aconseja el empleo de benzodiazepinas de larga duración, opiodes o antipsicóticos como medicamentos de primera línea. El empleo de melatonina o agonistas como ramelteon ha sido sustentado en algunos estudios. Antidepresivos sedantes como clomipramina, mirtazapina, amitriptilina o trazodone podrían usarse con precaución en la medida que la depresión sea una condición asociada. Es muy discutible su administración con fines exclusivamente hipnóticos. Los antihistamínicos no son recomendados en insomnio crónico y la evidencia para valeriana no es del todo concluyente ${ }^{(32)}$.

Tabla 2. Higiene del sueño en el adulto mayor*

a. Mantener una misma hora de despertar y número de horas de sueño.

b. Disminuir o eliminar las siestas, se permiten siestas vespertinas menores de 30 minutos si no afectan el sueño nocturno.

c. Realizar ejercicio regular, pero no inmediatamente antes de dormir.

d. Usar la cama solo para dormir o tener relaciones sexuales.

e. Evitar comer inmediatamente antes de dormir.

f. Limitar o eliminar el alcohol, cafeína y nicotina particularmente antes de dormir.

g. Mantener una rutina de preparación antes de ir a la cama (por ejemplo, el aseo, lectura).

h. Controlar el ambiente nocturno con una temperatura, tranquilidad y oscuridad confortable.

i. Vestir ropa holgada y confortable para dormir.

j. Si no concilia el sueño en $30 \mathrm{~min}$, levantarse de la cama y realizar actividades relajantes como escuchar música o leer, pero sin exponerse a luz intensa.

*Principios de Geriatría y Gerontología. 2da Ed: Lima; Universidad Peruana Cayetano Heredia; 2011
El manejo racional del insomnio en el adulto mayor involucraría entonces: 1) identificación de la causa, su corrección y la de eventuales trastornos comórbidos. 2) la posibilidad del abordaje a través de terapia cognitivo-conductual y 3) la complementariedad con medicación por tiempo definido.

Trastornos del ritmo circadiano. El adelanto de la fase de sueño que pudiera ser observado en algunos adultos mayores, puede ser manejado con la aplicación de luz vespertina. En pacientes con deterioro cognitivo se ha postulado el uso de melatonina y el empleo pautado de luz ${ }^{(32)}$.

Somnolencia diurna excesiva. La prevalencia de somnolencia diurna excesiva es $15,4 \%{ }^{(34)}$ Causas en el AM son privación de sueño, problemas de higiene de sueño, depresión, trastornos ocultos como SAOS o síndrome de piernas inquietas , trastornos metabólicos como hipotiroidismo, hipocortisolismo , hiponatremia, encefalopatía hepática, renal o insuficiencia cardiaca, entre otros.

Síndrome de apnea obstructiva del sueño (SAOS). La prevalencia estimada de SAOS, es de 3 a $32 \%$ para adultos en edad media y hasta $70 \%$ para el AM; se destaca un alto nivel de subdiagnóstico ${ }^{(35)}$.

El diagnóstico requiere una entrevista (Tabla 3) y una polisomnografía o, en algunos casos, una poligrafía cardiorrespiratoria. Síntomas habituales de SAOS son ronquido, pausas en la respiración, somnolencia diurna, insomnio o cansancio al despertar. Sin embargo, la sensibilidad diagnóstica, de ronquido, somnolencia diurna y sobrepeso es baja en el AM. En contraposición, el detrimento cognitivo, una mayor tendencia a las caídas y nicturia se agregan en la sospecha diagnóstica. Algunos relativizan el impacto de SAOS en el AM por un efecto de supervivencia y resiliencia. Contra ese argumento, la mortalidad cardio y cerebrovascular asociada a SAOS en AM es significativamente mayor en pacientes no tratados ${ }^{(36)}$. Una de cada tres personas con hipertensión arterial tiene SAOS, la hipertensión arterial resistente está asociada en $75 \%$ con SAOS. EI $50 \%$ de los pacientes con fibrilación auricular tiene SAOS ${ }^{(37)}$. SAOS severo no tratado se asocia con un riesgo tres veces mayor de ictus y un riesgo de enfermedad coronaria de 1,5 a 2 para este evento. Se estima que el $30 \%$ de diabéticos pueden tener SAOS ${ }^{(37)}$.

El tratamiento debería ofrecerse con un índice de apnea-hipopnea > 20/hora, particularmente cuando existe alguna comorbilidad como hipertensión arterial, diabetes, dislipidemia, antecedente de accidente cerebrovascular, infarto agudo de miocardio, arritmias cardiacas o deterioro cognitivo ${ }^{(38)}$.

Para los casos severos, el CPAP (presión positiva de aire sobre la vía aérea) es la mejor opción terapéutica. 
Tabla 3. Evaluación clínica de problemas de sueño en el adulto mayor

1. Cuestionarios: pueden ser administrados en la sala de espera

a. Escala de Pittsburgh

b. Escala de Epworth

2. Preguntas de cribaje planteadas en todo paciente

a. ¿Tiene dificultades para iniciar o mantener el sueño?

b. ¿Despierta cansado por la mañana independientemente de las horas que duerme?

c. ¿Está fatigado, cansado, tiene problemas con la memoria o le cuesta concentrarse en el día?

d. ¿Ronca Ud. o ha roncado fuerte en el pasado?

e. ¿Su sueño se interrumpe para ir a orinar?

f. ¿Tiene problemas con el equilibrio o ha tenido caídas?

3. Historia de sueño: se recomienda sea hecha en conjunción con cohabitantes o miembros de la familia

a. Horarios de alimentos, sueño y medicación.

b. Somnolencia diurna

c. Despertares nocturnos y su causa aparente

d. Ronquidos o pausas en la respiración.

e. Evaluar higiene de sueño.

f. Antecedentes médicos

4. Plan de trabajo

a. Diario y bitácora de sueño

b. Exámenes de laboratorio

c. Test fisiológico de sueño: actigrafía, polisomnografía o poligrafía cardiorrespiratoria

5. Tratamiento

a. Terapia no farmacológica: terapia cognitivo conductual, CPAP/BiPAP

b. Terapia farmacológica

CPAP: siglas en inglés de "presión positiva de aire sobre la vía aérea" BiPAP: siglas en inglés de "sistema de bipresión positiva"

Creado por Dr. Darwin Vizcarra Escobar

La adherencia al tratamiento se ve un tanto afectada en AM (38) requiriendo una mayor participación de los cuidadores. Estas dificultades no descalifican la necesidad del tratamiento.

Los dispositivos de avance mandibular se reservan para SAOS leve, o si existiera intolerancia al CPAP. Algunos casos individualizados pudieran ser beneficiados por cirugía maxilofacial o de la vía aérea superior. El empleo de electrodos implantables que estimulan el músculo hipogloso es una línea en desarrollo. El control estricto de la posición pudiera beneficiar a entre 10 a $15 \%$ de los pacientes ${ }^{(39)}$. El control del sobrepeso y del consumo de alcohol, tabaco o sedantes es importante, pero no sustituye al manejo señalado para los casos severos.
Síndrome de piernas inquietas(SPI). EISPI es considerado el desorden de movimiento más frecuente, aumenta con la edad y afecta entre el 1,9 al $15 \%$ de la población ${ }^{(40)}$. En el AM se identifican dos fenotipos: 1) con inicio antes de los cincuenta años, usualmente de origen genético. 2) con inicio después de los cincuenta años, usualmente secundario a algún trastorno por investigar. Existe asociación con otras condiciones tales como deficiencia de hierro o problemas renales. El cuadro se caracteriza por la necesidad de mover las piernas, que aparece en el reposo y con predominio nocturno, en tanto que el movimiento voluntario alivia transitoriamente esta sensación. Es necesario descartar patologías como polineuropatías o calambres. El diagnóstico es esencialmente clínico y un $80 \%$ de las personas con esta condición pueden tener movimientos periódicos involuntarios durante el sueño. EI SPI debe considerarse entre las causas de insomnio y somnolencia. Estudios recientes proponen un incremento del riesgo vascular en SPI. El tratamiento dependerá del nivel de severidad. Los medicamentos más empleados son los agonistas dopaminérgicos como pramipexole, ropirinole, rotigotine y los gabapentinoides.

Trastorno de comportamiento asociado a la fase de sueño REM (RBD). Se produce por la pérdida de la inhibición fisiológica del tono muscular del sueño REM. El paciente, usualmente, actúa los sueños, generalmente hiperdinámicos o violentos, relacionados con animales o riñas. Se describe en asociación con alfasinucleinopatías, (Parkinson, enfermedad por cuerpos de Lewy y atrofia de múltiples sistemas). El RBD es mucho menos frecuente en pacientes con enfermedad de Alzheimer. Los tratamientos de elección son melatonina y clonazepan.

En conclusión, los trastornos de sueño en el AM son frecuentes, importantes, tratables, pero subdiagnosticados. La principal tarea, aún pendiente, está en su reconocimiento.

Contribuciones de autoría: TTR, RA, DVE han participado conjuntamente en la concepción del artículo, su redacción y aprobación de la versión final. TTR redactó el subtema trastornos neurocognitivos mayores, RA redactó el subtema trastornos afectivos en el adulto mayor y DVE redactó trastornos del sueño en el adulto mayor.

Fuentes de financiamiento: autofinanciado.

Conflictos de interés: los autores declaran no tener conflictos de interés.

\section{REFERENCIAS BIBLIOGRÁFICAS}

1. De Mendonça Lima CA, Ivbijaro G. Mental health and wellbeing of older people: opportunities and challenges. Mental Health Fam Med. 2013;10(3):125-7.

2. American Psychiatric Association. Diagnostic and Statistical Manual of Mental Disorders, $5^{\text {th }}$. Edition. DSM-5. Washington DC: American Psychiatric Association; 2013.

3. Nowrangi MA, Rao V, Lyketsos CG. Epidemiology, assessment and treatment of dementia. Psychiatr Clin North Am. 2011;34(2):275-94. doi: 10.1016/j.psc.2011.02.004

4. Cano C. El anciano con demencia En: Abizanda P, Rodríguez L. Tratado de Medicina Geriátrica. Fundamentos de la atención sanitaria a los mayores. Madrid: Elsevier; 2015. p. 478-82. 
5. Prince M. World Alzheimer's Report 2009. London: Alzheimer's Disease International; 2009.

6. Dacks PA, Andrieu S, Blacker D, Carman AJ, Green AM, Grodstein F, et al. Dementia Prevention: optimizing the use of observational data for personal, clinical, and public health decision-making. J Prev Alzheimers Dis. 2014;1(2):117-23.

7. Libre Rodríguez JJ, Ferri CP, Acosta D, Guerra M, Huang Y, Jacob KS, et al. Prevalence of dementia in Latin America, India, and China: a population-based cross-sectional survey. Lancet. 2008;372(9637):46474 . doi: 10.1016/S01406736(08)61002-8.

8. Custodio N, García A, Montesino R, Escobar J, Bendezú L. Prevalencia de demencia en una población urbana de Lima-Perú: estudio puerta a puerta. An Fac med. 2008;69(4):233-8.

9. Instituto Nacional de Salud Mental. Estudio epidemiológico de Salud mental en Lima metropolitana y Callao-Replicación 2012. Informe General. Anales de Salud Mental, 2013; XXIX(Supl 1):1-392.

10. González P, Buuonantte F, Cáceres M. Del deterioro cognitivo leve al trastorno neurocognitivo menor: avances en torno al Constructo. Neurol Arg. 2015;7(1):51-8. doi: 10.1016/j. neuarg.2014.08.004

11. Tsoi KK, Chan JY, Hirai HW, Wong SY, Kwok TC. Cognitive Tests to Detect Dementia: A Systematic Review and Meta-analysis. JAMA Intern Med. 2015;175(9):1450-8. doi: 10.1001/ jamainternmed.2015.2152.

12. Custodio N, García A, Montesinos R, Lira D, Bendezú L. Validación de la prueba de dibujo del reloj - versión de manos - como prueba de cribado para detectar demencia en una población adulta mayor de Lima, Perú. Rev Peru Med Exp Salud Publica. 2011;28(1):2934.

13. AGS Choosing Wisely Workgroup. American Geriatrics Society identifies another five things that healthcare providers and patients should question. J Am Geriatr Soc 2014;62(5):950-60. doi: 10.1111 /jgs.12770.

14. Casey DA. Management of the patient in Geriatric Psychiatry. In: Tasman A, Kay J, Lieberman JA, First MB, Riba $M B$, editors. Psychiatry. $4^{\text {th }}$ ed. Vol. 2. Oxford: Wiley Blackwell; 2015. p. 2439-52.
15. Fountoulakis KN, O’Hara R, Iacovides A, Camilleri CP, Kaprinis S, Yesavage J. Unipolar late-onset depression: A comprehensive review. Ann Gen Hosp Psychiatry. 2003;2(1):11.

16. Yuen GS, Gunning FM, Woods E, Kilmstra SA, Hoptman MJ, Alexopoulos GS. Neuroanatomical correlates of apathy in late-life depression and antidepressant treatment response. J Affect Dis. 2014;166:179-86. doi: 10.1016/j. jad.2014.05.008.

17. Charney DS, Reynolds CF 3rd, Lewis L, Lebowitz BD, Sunderland T, Alexopoulos GS, et al. Depression and Bipolar Support Alliance Consensus Statement on the unmet needs in diagnosis and treatment of mood disorders in late life. Arch Gen Psychiatry. 2003;60(7):664-72.

18. Steffens DC, Skoog I, Norton MC, Hart AD, Tschanz JT, Plassman BL, et al. Prevalence of depression and its treatment in an elderly population. The Cache County Study. Arch Gen Psychiatry. 2000;57(6):601-7. doi:101001/pubs.Arch Gen PsychiatryISSN-0003-990x-57-6-yoa9308.

19. Lavretsky H, Kuman A. Clinically significant non-major depression: old concepts, new insights. Am J Geriatr Psychiatry. 2002;10(3):239-55.

20. Raue PJ, Ghesquiere AR, Bruce ML. Suicide risk in primary care: identification and management in older adults. Curr Psychiatry Rep. 2014;16(9):466-70. doi: 10.1007/ s11920-014-0466-8.

21. Guerra M, San Martín JC. Depresión en la Adultez Mayor. En: Alarcón RD, Mazzotti G, Nicolini H, editors. Psiquiatría. $3^{a}$ ed. Washington DC: Organización Panamericana de la Salud; 2012. p. 837-51.

22. Taylor WD, Aizenstein HJ, Alexopoulos GS. The vascular depression hypothesis: mechanisms linking vascular disease with depression. Mol Psychiatry. 2013;18(9):963-74. doi: 10.1038/ mp.2013.20.

23. Sajatovic M, Strejilevich SA, Gildengers AG, Dols A, Al Jurdi RK, Forester BP, et al. A Report on older-age bipolar disorder from the International Society for Bipolar Disorders Task Force. Bipolar Disord. 2015;17(7):689704. doi: 10.1111/bdi.12331.

24. Shulman K, Tohen M, Satlin A, Mallya G, Kalunian D. Mania compared with unipolar depression in old age. Am J Psychiatry. 1992;149(3):341-5.

25. Frank C. Pharmacologic treatment of depression in the elderly. Can Fam Physician. 2014;60(2):121-6.

26. Kiosses DN, Ravdin LD, Gross JJ, Raue P, Kogbi N, Alexopulos GS. Problem adaptation therapy for older adults with major depression and cognitive impairment: a randomized clinical trial. JAMA Psyhiatry. 2015;72(1):22-30. doi: 10.1001/ jamapsychiatry.2014.1305.

27. Verrusio W, Andreozzi P, Marigliano B, Renzi A, Gianturco V, Pecci MT, et al. Exercise training and music therapy in elderly with depressive syndrome: a pilot study. Complement Ther Med. 2014;22(4):614-20. doi: 10.1016/j. ctim.2014.05.012.

28. Dybedal GS, Tanum L, Sunde K, Gaarden TL, Bjolseth TM. Cognitive side-effects of electroconvulsive therapy in elderly depressed patients. Clin Nduropsychol. 2014;28(7):1071-90. doi: 10.1080/13854046.2014.958536.

29. Brunoni AR, Boggio PS, De Raedt R, Benseñor IM, Lotufo PA, Namur V, et al. Cognitive control therapy and transcranial direct current stimulation for depression: a randomized, doubleblinded, controlled trial. J Affect Disord. 2014;162:43-9. doi: 10.1016/j. jad.2014.03.026.

30. Alhola P, Polo-Kantola P. Sleep deprivation: impact on cognitive performance. Neuropsychiatr Dis Treat. 2007;3(5):553-67.

31. Lal C, Strange C, Bachman D. Neurocognitive impairment in obstructive sleep apnea. Chest. 2012;141(6):1601-10. doi: 10.1378/ chest.11-2214.

32. Urrestarazu E, Iriarte J. Clinical management of sleep disturbances in Alzheimer's disease: current and emerging strategies. Nature Sci Sleep. 2016;8:21-33. doi: 10.2147/NSS. S76706.

33. Doghramji K. The epidemiology and diagnosis of insomnia. Am J Manag Care. 2006;12(8 Suppl):S214-S20.

34. Vashum KP, McEvoy MA, Hancock SJ, Islam MR, Peel R, Attia JR, et al. Prevalence of and associations with excessive daytime sleepiness in an Australian older population. Asia Pac J Public Health. 2015;27(2):NP227584. doi: 10.1177/1010539513497783. 
35. Tufik S, Santos-Silva R, Taddei JA, Bittencourt LR. Obstructive Sleep Apnea Syndrome in the Sao Paulo Epidemiologic Sleep Study. Sleep Med. 2010;11(5):441-6. doi: 10.1016/j. sleep.2009.10.005.

36. Martínez-García MA, CamposRodríguez F, Catalán-Serra $\mathrm{P}$, SolerCataluña JJ, Almeida-Gonzalez C, De la Cruz Morón I, et al. Cardiovascular mortality in obstructive sleep apnea in the elderly: role of long-term continuous positive airway pressure treatment: a prospective observational study. Am J Respir Crit Care Med. 2012;186(9):909-16. doi: 10.1164/ rccm.201203-0448OC.

37. Somers VK, White DP, Amin R, Abraham WT, Costa F, Culebras A, et al. Sleep apnea and cardiovascular disease: an American Heart Association/
American College of Cardiology Foundation Scientific Statement from the American Heart Association Council for High Blood Pressure Research Professional Education Committee, Council on Clinical Cardiology, Stroke Council, and Council On Cardiovascular Nursing. In collaboration with the National Heart, Lung, and Blood Institute National Center on Sleep Disorders Research (National Institutes of Helath). Circulation. 2008;118(10):1080-111. doi: 10.1161/ CIRCULATIONAHA.107.189375.

38. Ou Q, Chen YC, Zhuo SQ, Tian XT, $\mathrm{He} \mathrm{CH}, \mathrm{Lu} \mathrm{XL}$, et al. Continuous Positive Airway Pressure Treatment Reduces Mortality in Elderly Patients with Moderate to Severe Obstructive Severe Sleep Apnea: A Cohort Study. PLoS One. 2015;10(6):e0127775. doi: 10.1371/journal.pone.0127775.
39. Bignold, JJ, Deans-Costi G., Goldsworthy MR, Robertson CA, McEvoy D, Catcheside PG, et al. Poor long-term patient compliance with the tennis ball technique for treating positional obstructive sleep apnea. J Clin Sleep Med. 2009;5(5):428-30.

40. Ohayon MM, O'Hara R, Vitello MV. Epidemiology of restless legs syndrome: a synthesis of the literature. Sleep Med Rev. 2012;16(4):283-95. doi: 10.1016/j.smrv.2011.05.002.

Correspondencia: Tania Tello Rodriguez Dirección: Instituto de Gerontologia, Universidad Peruana Cayetano Heredia. Av. Honorio Delgado 430, Urb. Ingeniería, San Martin de Porres. Lima, Perú

Teléfono: (+511) 993489835 / Telefax: 3190007 Correo electrónico: tania.tello.r@upch.pe

\section{Suscríbete a nuestro canal de YOlltuhe y disfruta la mejor y más completa información de investigación científica} www.youtube.com/RPMESP

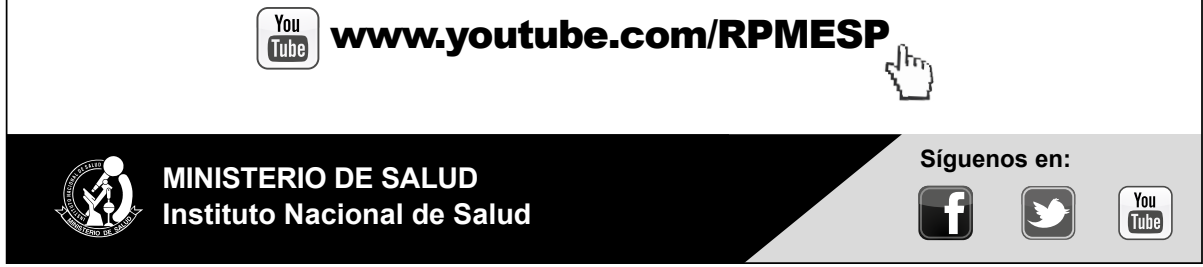

